\title{
Las políticas universitarias del nuevo gobierno en Argentina: el desafío de fortalecer la democratización de la Universidad
}

\author{
Anabella Celeste LuCARdi
}

Magíster en Administración y Políticas Públicas (Universidad de San Andrés), abogada (Universidad de Buenos Aires), doctoranda en Ciencias Sociales (Universidad de Buenos Aires), directora de la carrera de abogacía de la Universidad Nacional de Avellaneda, docente e investigadora de la misma universidad.

Resumen

El presente artículo pretende analizar los anuncios sobre las potenciales líneas de políticas públicas para la educación superior que implementará el gobierno que se inauguró en diciembre de 2019 en Argentina, y establecer posibles continuidades con la direccionalidad de las políticas públicas universitarias que tuvieron lugar en el país entre 2003 y 2015. Concretamente se aborda la noción de democratización de la universidad y del acceso al conocimiento presente en las pautas de las políticas para el sector expresadas por el nuevo gobierno; así como también, en las razones que fundaron la política pública de expansión del sistema universitario de gestión estatal que tuvo lugar en Argentina entre 2003 y 2015.

Palabras clave: políticas públicas universitarias; políticas públicas de Educación Superior; democratización universitaria; derecho a la Universidad; Ley de Educación Superior; expansión del sistema universitario. 


\title{
As políticas universitárias do novo governo na Argentina: o desafio de fortalecer a democratização da Universidade
}

\section{Resumo}

O presente artigo analisa o anúncio das potenciais linhas de políticas públicas na Educação Superior que vão se implementar no governo inaugurado na Argentina em dezembro de 2019, e estabelece possíveis continuidades com a direcionalidade das políticas públicas universitárias que tiveram lugar no país entre 2003 e 2015. Concretamente, se aborda a noção de democratização da universidade e o acesso ao conhecimento presente nas políticas para o sector que se expressam no novo governo, assim como as razões nas que se fundou a política pública de expansão do sistema universitário de gestão estadual que teve lugar na Argentina entre 2003 e 2015.

Palavras-chave: políticas públicas universitárias; políticas públicas de Educação Superior; democratização universitária; direito à universidade; Lei de Educação Superior; expansão do sistema universitário.

\section{The New Government's University Policies in Argentina: The Challenge of Strengthen the Democratization of Uni- versity}

\begin{abstract}
This research examines the potential public policies for the higher education that the new government, which began in December 2019, will introduce. Other objective of the study is to establish the possible ongoing actions concerning the university public policies that took place in Argentina between 2003 and 2015. In particular, the article addresses the notion of the university democratization and the access to the current knowledge of policies for this educational sector, that is manifested by the new government, as well as the reasons that founded the public policy of the university system expansion in the Argentina's government management between 2003 and 2015.
\end{abstract}

Keywords: university public policies, higher education public policies, university democratization, university right, higher education law, university system expansion. 


\section{Presentación}

Los contornos del sistema universitario de gestión estatal en Argentina permiten reconocer la coexistencia de instituciones cuya creación data de cuatrocientos años atrás con universidades creadas muy recientemente. Esta afirmación da cuenta de su notable expansión desde los albores de la fundación de la Universidad Nacional de Córdoba en 1613 hasta la actualidad. El proceso expansivo fue lento hasta 1960, mientras que a partir de la década del setenta fue muy vigoroso, pues desde entonces se produjeron incrementos significativos en la cantidad de instituciones educativas universitarias en tres momentos determinados. Entre 1971 y 1973 tuvo lugar la primera oleada expansiva, con la creación de más de una decena de nuevas universidades. Posteriormente la segunda oleada expansiva data de los años noventa: entre 1989 y 1995 se crearon nueve instituciones educativas universitarias. Finalmente a partir del 2003 y hasta el 2015, asistimos a un nuevo periodo expansivo del sistema universitario de gestión estatal, que se manifestó con la creación de veintitrés universidades nacionales e institutos universitarios nacionales. El cambio de gobierno en 2015 en Argentina puso en suspenso las políticas de expansión del sistema universitario estatal y produjo un retroceso en general en las políticas orientadas al desarrollo de la educación superior, la ciencia y la tecnología. Concretamente, el presupuesto universitario cayó más de $40 \%$ y su incremento real no logró equiparar los niveles de inflación. Por su parte, la participación del gasto en educación superior en el Producto Interno Bruto (PIB) también sufrió una fuerte retracción. La inversión destinada a las universidades representó $0.7 \%$, en promedio, del PIB entre 2016 y 2019, por debajo del punto porcentual que había alcanzado en 2015. En contraste el nuevo gobierno que se inauguró en Argentina, en diciembre de 2019, declara observar un lugar de privilegio para las universidades y el sistema científico tecnológico y un interés por la reconstrucción de los ámbitos ligados a la producción de conocimiento. Entre las primeras acciones del actual presidente, Alberto Fernández, se encuentra la jerarquización de la cartera de Ciencia y Tecnología, que volvió a tener rango de ministerio, el impulso a la investigación con la duplicación de los ingresos de personal científico al Consejo Nacional de Investigaciones Científicas y Técnicas (CONICET), organismo estatal que concentra dicha función, y un incremento en el financiamiento de las becas y subsidios para proyectos de investigación científica y tecnológica.

Volviendo a la cuestión universitaria, y aun cuando los escasos meses transcurridos desde el inicio del nuevo gobierno no permiten más que aproximaciones a la línea que adoptarán las políticas públicas para la educación superior, nos interesa considerar especialmente los anuncios que en esta materia hizo el presidente frente al Parlamento, ello en la medida que sus directrices en materia de políticas públicas para la universidad hilvanan y parecieran mostrar una continuidad con la direccionalidad de las políticas universitarias que se desarrollaron en Argentina entre 2003 y 2015, durante los tres periodos de gobierno del mismo signo político que el actual, que tuvieron como presidentes a Néstor C. Kirchner y a Cristina Fernández de Kirchner, la actual vicepresidenta. 
El primero de los anuncios se relacionó con la necesidad de iniciar el debate sobre una nueva Ley de Educación Superior que permita fortalecer el sistema universitario, con la gratuidad y el acceso irrestricto como puntos de partida. Mientras que el segundo hizo hincapié en la necesidad de democratizar el acceso al conocimiento y fortalecer el vínculo entre la universidad, la comunidad y el sector productivo, para garantizar el desarrollo territorial. El aludido concepto de democratización expresa una idea muy desarrollada en la investigación sobre educación superior y por ello, en las próximas páginas, trataremos de reflexionar sobre el sentido que adoptó la noción de democratización en el contexto de la última ola expansiva del sistema universitario de gestión estatal en Argentina entre 2003 y 2015, y que tuvo impacto especialmente en el conurbano bonaerense, la segunda región más densamente poblada de Argentina después de la Ciudad de Buenos Aires, donde se concentran aproximadamente 10 millones de habitantes.

Nuestra hipótesis considera que la política pública de expansión del Sistema de Educación Superior Universitario de Gestión Estatal en Argentina entre 2003 y 2015 estuvo caracterizada por un discurso sobre la "democratización" de la universidad, presente fundamentalmente en las razones declaradas por los actores que impulsaron el mencionado esquema y que se plasmó también en los diversos documentos fundacionales de estas instituciones. Este discurso motivó también la formulación de otras políticas en el contexto universitario, incluida una reforma parcial de la Ley de Educación Superior, y por dicho motivo creemos que caracterizó en general a las actividades públicas relacionadas con la educación universitaria durante el periodo. En relación con ello, y en el escenario actual, el nuevo gobierno, que comenzó a dar sus primeros pasos en el contexto de un planeta convulsionado por el avance de una enfermedad pandémica, recupera la idea de democratización, lo que exige volver a revisarla y redimensionarla.

En este artículo reflexionaremos sobre la idea de democratización universitaria, que exige concebir a la universidad como un derecho. El derecho a la educación superior cuenta en Argentina reconocimiento legal desde 2015, a partir de la señalada reforma de la Ley de Educación Superior. A su vez, nos detendremos en la política expansiva del Sistema Universitario de Gestión Estatal que tuvo lugar entre 2003 y 
2015, dado que garantizar el ejercicio del derecho a la universidad requiere de una serie de condiciones entre las cuales se cuenta la existencia de un mayor número y de una mejor distribución geográfica de las instituciones universitarias encargadas de posibilitarlo.

\section{Las razones declaradas de la política pública de creación de universidades. El análisis de los documentos fundacionales de un conjunto de universidades nuevas del conurbano bonaerense}

En 2001 se produjo en Argentina una crisis del Estado neoliberal que implicó también la debacle del modelo de acumulación ligado a la valorización financiera, explicado por las teorías políticas asociadas al neoliberalismo económico que impulsaron los gobiernos de la mayoría de los países latinoamericanos durante la década de los noventa. Producto de esta crisis, sobrevino en Argentina una etapa de incesantes luchas políticas y sociales por la fijación de un nuevo patrón de acumulación. En este contexto, se observó la reconfiguración de una naciente forma de Estado, tributaria de las tradiciones nacionales y populares que, a través del despliegue de la administración estatal activa, orientada en una perspectiva neodesarrollista, puede caracterizarse como un proceso populista de democratización, con influencias democráticas y republicanas, inserto en la línea de experiencias del mismo tipo en la región (Sidicaro, 2003; Basualdo, 2006 y 2011; Varesi, 2010 y 2011; CENDA, 2010; Cantamutto, 2013; Laguado y Cao, 2015).

En este sentido, a partir de 2003 y durante los tres periodos de gobierno siguientes, se puso en marcha una batería de actividades públicas sociales cuyo objetivo fue mejorar los niveles de inclusión social. Entre ellas se destaca la Asignación Universal por Hijo, que, junto con acciones públicas de tipo educativas como las que impulsaron la Ley de Financiamiento Educativo y la Ley de Educación Nacional, en un contexto económico que permitió gradualmente la recuperación del empleo y la recomposición de una escena social marcada por los grandes índices de pobreza, redundaron progresivamente en una ampliación de derechos para los sectores sociales más postergados. En este marco tuvo lugar la implementación de una actividad orientada a ampliar las dimensiones del sistema universitario, caracterizada por la bibliografía especializada como la tercera ola expansiva del sistema universitario de gestión estatal en nuestro país (Chiroleu, 2012; Chiroleu y Iazzetta, 2012; Ezcurra, 2011; Pérez Rasetti, 2012; Rinesi, 2015; Suasnábar y Rovelli, 2012, Unzué, 2016).

Analizaremos a continuación la política de expansión del sistema universitario de gestión estatal en Argentina, particularmente a partir del estudio de las razones que declara la creación de universidades en el conurbano bonaerense, territorio donde se concentró una porción muy significativa de las nuevas instituciones universitarias.

Para ello practicaremos una exhaustiva revisión de las leyes de creación de las instituciones educativas, de sus proyectos institucionales, de los dictámenes de la Comisión Nacional de Evaluación y Acreditación Universitaria (CONEAU) previos a la puesta en funcionamiento de las nuevas 
instituciones universitarias y de los estatutos de las propias universidades, con el objetivo de dar cuenta de las motivaciones que orientaron la formulación de estas actividades. Corresponde señalar que en el proceso de la política pública que posibilitó la expansión geográfica del sistema universitario, participaron diversos actores y ello naturalmente implica que las razones declaradas sean múltiples. La creación de instituciones educativas universitarias constituye un acto complejo en el cual intervienen, en primer lugar, sucesiva y complementariamente, dos poderes de gobierno (Ejecutivo y Legislativo). De modo que las razones que declara en primer lugar la política pública, y que observamos en las leyes de creación, expresan consensos a los que pudieron arribar los impulsores de distintos proyectos de ley en cada una de las Cámaras del Congreso de la Nación, que por su parte, fueron legisladores pertenecientes al partido del gobierno. A su vez, las razones que surgen de los instrumentos fundacionales de las nuevas universidades (proyectos institucionales, dictámenes de la CONEAU y estatutos), dan cuenta de la participación de nuevos y distintos actores: a) los rectores normalizadores que conducen el proceso de organización de las instituciones universitarias y que fueron designados por el Poder Ejecutivo y las autoridades universitarias que los acompañan en dicha tarea en cada una de las nuevas universidades, b) el Ministerio de Educación, que tiene a su cargo la facultad de autorizar el funcionamiento de las universidades creadas conforme a la viabilidad de los proyectos institucionales que éstas formulen y la prerrogativa de aprobar sus estatutos provisorios y c) la CONEAU, organismo descentralizado que opera en el marco de la cartera educativa y que le corresponde emitir dictamen previo a la autorización que otorga el Ministerio de Educación.

En primer lugar, cabe señalar que la Universidad Nacional de Avellaneda (UNDAV), la Universidad Nacional del Oeste (UNO), la Universidad Nacional de Moreno (UNM), la Universidad Nacional Arturo Jauretche (UNAJ) y la Universidad Nacional de José Clemente Paz (UNPAZ) fueron creadas por leyes del Congreso de la Nación, sancionadas en 2009, que llevan los números 26.543, 26.544, 26.575, 26.576 y 26.577, respectivamente. Con posterioridad a su creación legal, se designaron en cada una de ellas, a instancias del Ministerio de Educación, rectores organizadores para promover y conducir el proceso de formulación del proyecto institucional y el estatuto provisorio. Los proyectos institucionales fueron puestos a consideración de la CONEAU que emitió sendos dictámenes favorables en respuesta a la solicitud de puesta en marcha de las mencionadas instituciones universitarias.

Las leyes de creación de las distintas universidades aquí consideradas utilizan estructuras similares y su articulado es más bien sintético. Las leyes de creación de la UNDAV y de la UNO contienen alusiones semejantes en relación con los objetivos de la institución que se crea, y resaltan que la oferta académica no se superpondrá a la de otras instituciones educativas de la región donde se emplazan. Asimismo plantean la adecuación de los programas y las currículas de estudio en un perfil específico vinculado con las características socioeconómicas y culturales del distrito en particular y de la región en general. En el caso de la UNDAV, la ley incluye una enumeración no taxativa de las áreas en torno a las cuales se orientará la oferta aca- 
démica, siendo éstas las Artes, el Medio Ambiente, el Deporte, la Producción y el Trabajo. En el caso de la UNO, señala que se promoverán carreras orientadas al desarrollo económico y social, con acento en aquellas vinculadas con la producción de bienes y servicios, como así también de las ciencias ambientales. Por su parte, las leyes de creación de la UNAJ y la UNPAZ descartan la referencia de los objetivos generales de la creación de la universidad y no aluden a las características de su oferta académica. Por último la ley de creación de la UNM plantea la necesidad de promover social, económica y culturalmente la zona de asiento y el área de influencia de la universidad.

Los proyectos institucionales constituyen documentos que abordan de manera integral la justificación institucional y académica de las nuevas instituciones educativas para su evaluación por parte de la CONEAu y el Ministerio de Educación. Por su parte los estatutos -además de contener principalmente precisiones de carácter organizativo- cristalizan la visión, misión y valores en los que se funda la institución y describen sus fines y objetivos. En este sentido, nos interesa considerar los proyectos institucionales y los estatutos de las universidades en la medida en que estos arrojan información acerca de las motivaciones que rodearon la puesta en marcha de estas instituciones. Desde la perspectiva de políticas públicas que adoptamos, se procura analizar si el propósito de favorecer la democratización de la universidad, como concepto, se encuentra presente en los idearios institucionales que las universidades plasmaron en sus respectivos proyectos institucionales y normas estatutarias, y adicionalmente, considerar cuál es la valoración de la CONEAU en torno a dichos planteamientos a través del análisis de los dictámenes correspondientes.

El estatuto académico de la UNDAV reconoce a la educación como un derecho humano universal, y como un bien público social, como un deber y político prioritario del Estado para construir una sociedad justa, y para fortalecer el desarrollo social y económico de la Nación. Asume los principios de equidad e igualdad de oportunidades de la educación pública. Asimismo, de su proyecto institucional se extrae que entre sus objetivos se encuentra el de propender a la formación conforme a criterios de equidad, excelencia, compromiso social y desarrollo ciudadano y el de propiciar el desarrollo y mejorar la calidad de vida de la comunidad en la que se inserta. 
El estatuto provisorio de la UNO, por su parte, concibe a la educación superior como un derecho humano y un bien público de decisiva transcendencia social. En ese contexto señala también como misión la de adecuarse a las necesidades locales y regionales. En este caso, no obstante pronunciarse en forma favorable a la solicitud de puesta en marcha de la UNO, el informe de la CONEAU realiza un conjunto de recomendaciones dirigidas al rector organizador, orientadas a resolver las debilidades institucionales que plantea el proyecto.

El proyecto institucional de la UNM considera que el crecimiento de la demanda de educación superior debe ser atendido por el Estado y que esta intervención persigue la democratización de las oportunidades de acceso y permanencia a estudios de nivel superior. Concretamente considera que en el conurbano bonaerense existen dificultades de acceso a la educación superior y que, por esta razón, la oferta de estudios universitarios de gestión estatal debe concentrarse en la región, con miras a obtener un impacto positivo sobre las desigualdades en el ingreso. Asimismo hace referencia a las características económicas del segundo cordón del conurbano bonaerense donde se inserta, en particular a las limitaciones del progreso de las fuerzas productivas, y afirma que el hecho de modificar la distribución de los saberes en la sociedad y elevar el nivel educativo del conjunto de la población posibilita un crecimiento económico sostenido. Adicionalmente expresa que la creación de una universidad de gestión estatal forma parte de una política de localización del gasto público que propenda a sentar las bases para un desarrollo social y productivo regionalmente armónico. Por último en su estatuto académico reconoce que la educación, en todos sus niveles, constituye un derecho humano universal y promueve la igualdad de oportunidades.

A su turno, el proyecto institucional de la UNAJ ubica el acento en la cuestión del territorio y la valorización de la cuestión local y practica una caracterización de la región comprendida por el segundo cordón del conurbano. En este contexto, afirma que, respecto de su área de influencia, la Universidad debe actuar como motor dinamizador, agente de desarrollo de políticas, como instrumento de creación de capital y como centro de formación permanente. Señala que la creación de universidades en el conurbano bonaerense contribuye con el logro de los compromisos de la Nación respecto del desarrollo humano en un área de profunda vulnerabilidad social. Por su parte, el estatuto provisorio da cuenta de la vocación por contribuir al desarrollo económico, social y cultural de la región, mejorar la calidad de vida y fortalecer los valores democráticos de la sociedad, a través de la producción y distribución de conocimiento y de innovaciones científico-tecnológicas. Hace hincapié en que su tarea hermenéutica y axiológica debe estar orientada a atender las demandas sociales. Finalmente también asegura la igualdad de oportunidades. 
Por último el proyecto institucional de la UNPAZ identifica la creación de nuevas instituciones universitarias como parte de un proceso general de cambios en el sistema de educación superior y, en este contexto, reconoce la responsabilidad del Estado en la promoción de la universalización del acceso a la educación superior. Señala a la propia institución como favorecedora de la democratización del sistema universitario a través de tres instancias: a) por la incorporación de los sectores sociales tradicionalmente más desfavorecidos en términos socioeconómicos, $b$ ) por medio del incremento de las capacidades de la economía por el valor agregado del uso intensivo de conocimiento científico tecnológico, y c) mediante la mejora de las condiciones sociales e institucionales para el pleno ejercicio de una ciudadanía fundada democráticamente. Su estatuto reconoce a la educación superior como un bien público y un derecho social, cuyo ejercicio resulta fundamental para el desarrollo económico, social y cultural de la región.

El estudio descriptivo de los documentos reseñados nos permite presentar los siguientes hallazgos. En primer lugar, los instrumentos distinguen el derecho a la educación superior como un derecho humano universal y un bien público social. Por otra parte, reconocen la responsabilidad del Estado como garante del ejercicio del derecho a la universidad y consideran que el impulso de creación de universidades en el conurbano bonaerense constituye una política orientada a la democratización de las oportunidades de acceso y permanencia en el nivel superior. En tercer lugar, todos los documentos validan entre los objetivos de las instituciones educativas propiciar el desarrollo de la región donde se insertan y contribuir a la mejora de la calidad de vida de las comunidades que las circundan, orientando el desarrollo de estrategias vinculadas con el conocimiento científico y tecnológico a la resolución de las problemáticas locales. Por último, evidencian el principio de igualdad de oportunidades. A modo de conclusión parcial, resulta claro que la aspiración a la democratización de la universidad se encuentra presente en los idearios institucionales de las nuevas universidades. La intención democratizadora de la política pública de creación de instituciones educativas universitarias en el conurbano bonaerense y la concepción que reconoce a la educación superior como un derecho universal tienen reflejo en las normas estatutarias.

En cuanto a la formulación de líneas de acción programáticas para la expansión del sistema de educación superior, en 2009 la Secretaría de Políticas Universitarias (SPU) dependiente de la cartera educativa, creó el Programa de Diagnóstico y Evaluación para la Expansión de la Educación Superior (Res. SPU 299/2009) y posteriormente, su continuación: el Programa de Expansión de la Educación Superior (Res. SPU 1368/2012). El primer programa tenía, entre otros objetivos, el de atender la demanda de ampliar la cobertura geográfica de la educación superior, aumentando los niveles de democratización y logrando la obtención de más y mejores graduados, mientras que el segundo impulsaba la creación de Centros Regionales de Educación Superior (CRES). No obstante estos programas tuvieron magro resultado (Pérez Rasetti, 2012). Puede concluirse que la formulación de la política pública de creación de instituciones educativas universitarias de gestión estatal durante este tercer periodo expansivo careció del previo análisis y consulta a los organismos de la administración pública nacional involu- 
crados en la planificación de sistema (Ministerio de Educación y SPU) y que intervinieron en la decisión factores exógenos. Sin embargo, la planificación de la política universitaria en general estuvo orientada fuertemente por la direccionalidad política que ya se observaba en las políticas públicas sociales de alcance general y sectorial para el sistema educativo que se impulsaron desde el Poder Ejecutivo. En ese contexto, la orientación de la política gubernamental universitaria durante el periodo fue promovida por la cabeza del Ejecutivo Nacional, con una fuerte referencia al aliento de la democratización universitaria, que se evidencia cuando se recorren las muy diversas piezas discursivas producidas sobre este asunto, en particular por la ex jefa de Estado Cristina Fernández de Kirchner ${ }^{1}$. En segundo lugar, intervino en su formulación el Poder Legislativo Nacional, a través del impulso parlamentario de los instrumentos correspondientes, principalmente, por parte de los representantes del partido de gobierno y concretamente mediante la sanción de las leyes de creación de universidades (con consensos alcanzados con otros sectores políticos o sin ellos) y el Poder Ejecutivo Nacional, no solo a través de la promulgación de las leyes correspondientes, sino por medio de la acción de los distintos órganos de la Administración Pública Nacional encargados de la ejecución de las políticas públicas universitarias.

\section{La Reforma de la Ley de Educación Superior: el derecho a la universidad en el centro de la escena}

En este punto, corresponde analizar el contenido de la reforma parcial de la Ley de Educación Superior (LES) que tuvo lugar en los últimos días del ciclo de gobiernos kirchneristas, en 2015. La reforma de la LES se produjo durante la presidencia de Cristina Fernández de Kirchner, actual vicepresidenta y a instancias del impulso de la Diputada Adriana Puigróss, quien, en el gobierno actual, detenta el cargo de viceministra de Educación de la Nación. Esta reforma reconoció que la educación y el conocimiento son un derecho humano personal y social, consagró la gratuidad, el ingreso irrestricto, y atribuyó al Estado la responsabilidad sobre la educación superior. Desde luego, la antigua Les sancionada en 1995, reflejaba el clima de la época, caracterizado por los compromisos con los organismos multilaterales de crédito y la mercantilización de la educación. Sin embargo, esta reforma parcial introdujo valiosas modificaciones que contribuyen a pensar en la universidad como un derecho, lo que resulta inherente a la idea de democratización. Dicho de otro modo, un concepto es presupuesto del otro. La democratización universitaria supone, en efecto, la existencia de un derecho a la educación superior. 
Concretamente, la Ley 27.204 de implementación efectiva de la responsabilidad del Estado en el nivel de educación superior sustituyó enteramente algunos artículos de la antigua LEs e incorporó otros. En primer lugar, reconoce que la educación y el conocimiento son un bien público y un derecho humano personal y social. En segundo lugar, ejerce una enumeración exhaustiva del contenido de la responsabilidad principal e indelegable del Estado Nacional, las provincias y la Ciudad Autónoma de Buenos Aires sobre la educación superior. Entre ellas, garantiza la igualdad de oportunidades y condiciones en el acceso, la permanencia, la graduación y el egreso; ordena proveer equitativamente, en la educación superior de gestión estatal, becas, condiciones adecuadas de infraestructura y recursos tecnológicos apropiados para todas aquellas personas que sufran carencias económicas verificables y asimismo dispone promover políticas de inclusión educativa. Como ya se señaló consagra la gratuidad de los estudios de grado en las instituciones educativas universitarias de gestión estatal y, en la misma línea de lo establecido por la Ley de Educación Nacional, prohíbe a las instituciones de educación superior de gestión estatal suscribir acuerdos o convenios con otros Estados, instituciones $\mathrm{u}$ organismos nacionales e internacionales públicos o privados, que impliquen ofertar educación como un servicio lucrativo o que alienten formas de mercantilización. Por último, reconoce el ingreso irrestricto a las carreras de grado, que complementa los procesos de nivelación y orientación profesional y vocacional no excluyentes que cada institución disponga. Como se observa, la reforma de la LES asigna al Estado la posición principal como planificador de las políticas educativas, desde una perspectiva que considera la educación un bien público y un derecho personal y social, posicionamiento que el presidente Fernández recupera cuando en su mensaje frente al Parlamento muestra además, preocupación por garantizar el cumplimiento efectivo de las leyes programáticas vigentes, con referencia a la Ley de Financiamiento Educativo, la Ley de Educación Nacional y la Ley de Educación Técnico Profesional.

Nos interesa, en este punto, reconstruir los antecedentes del enunciado legal del derecho a la educación superior en Argentina que emerge de la reforma parcial de la LES en 2015 pero en cuya prehistoria se reconocen una serie de acontecimientos. Tres de ellos ocurrieron a lo largo de la historia argentina, y

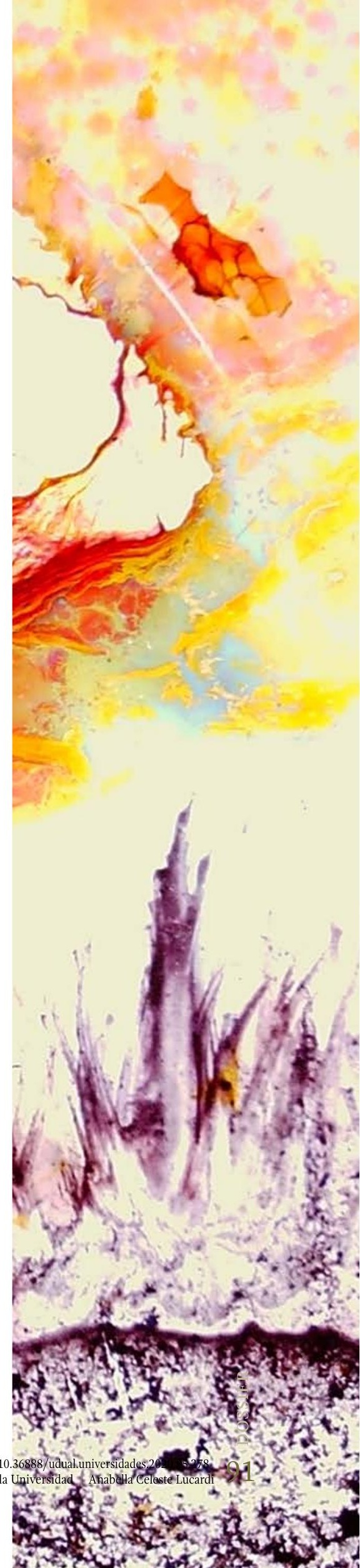


el cuarto tiene alcance regional y forma parte de un clima de época en cuyo contexto se comprueba una ampliación de derechos en toda América Latina. En primer lugar los sucesos vinculados con la Reforma Universitaria de 1918 y sus ecos posteriores en Latinoamérica. En segundo lugar, la masificación del acceso a la universidad a partir de la eliminación de los aranceles en 1949 y la instauración de la gratuidad universitaria durante el gobierno peronista. En tercer lugar, los tres procesos de expansión institucional del sistema universitario que reseñamos en la presentación de este artículo y que se desarrollaron, primero, en el marco del Plan Taquini, luego, durante los años noventa y finalmente, entre 2003 y 2015. En cuarto lugar, en el plano regional, la consagración de la educación superior como un derecho humano y universal, un bien público social y un deber del Estado en la Declaración Final de la Conferencia Regional de la Educación Superior de 2008 celebrada en Cartagena de Indias. Finalmente, recogiendo los antecedentes locales y regionales señalados, particularmente los más recientes, se produjo la reforma parcial en 2015 de la LES que, como ya mencionamos, atribuye al Estado la responsabilidad principal e indelegable en el nivel de educación superior en tanto la educación y el conocimiento son un bien público y un derecho humano personal y social.

En este contexto, el desafío del actual gobierno en Argentina pareciera ser el de proponer instrumentos legislativos que cristalicen políticas públicas que garanticen el efectivo ejercicio del derecho a la educación superior, para lograr que todas y todos tengan derecho a la universidad en condiciones de igualdad.

Una revisión de las investigaciones acerca del acceso a la educación superior y del ejercicio del derecho a la universidad en Argentina muestra que el mismo se encuentra fuertemente condicionado por el nivel de ingreso. En este sentido, las personas provenientes de los quintiles de menores ingresos participan en menor medida en la educación superior universitaria, en comparación con las personas pertenecientes a los quintiles de mayores ingresos. No obstante, la ejecución de políticas públicas para favorecer la ampliación del acceso al nivel universitario, pareciera permitir gradualmente una mayor representación de los sectores tradicionalmente excluidos del acceso a la educación universitaria, ampliando el ejercicio del derecho a la educación superior a un conjunto mayor de personas. De otro modo, la ausencia de políticas públicas que no posibiliten el ejercicio del derecho a la universidad por parte de los sectores más postergados, impactará negativamente en el efectivo ejercicio de ese derecho y evidenciará una menor representación de las personas cuyas familias poseen menores ingresos en la educación superior universitaria.

El debate sobre una nueva Ley de Educación Superior, anunciado por el Jefe de Estado, deberá considerar especialmente esta circunstancia. La preocupación por la democratización de la universidad y del acceso al conocimiento traduce una preocupación por la desigualdad. En contraste, el derecho a la universidad opera sobre el presupuesto de la igualdad. Ahora bien, en la medida en que la igualdad, en su plano real, no se concreta autónomamente, el rol del Estado y de las políticas públicas pareciera esencial 
para tornar posibles los escenarios de igualdad que exige la sociedad. En este contexto, el anuncio del nuevo gobierno acerca de sus intenciones de debatir una ley de educación superior que garantice la gratuidad y el acceso irrestricto a las universidades, y que democratice el acceso al conocimiento, se ubica en la línea de sentar bases legislativas fuertes que contribuyan a garantizar el ejercicio del derecho a la educación superior por parte de todos y todas quienes deseen acceder, permanecer y graduarse en la universidad.

\section{Reflexiones finales}

A modo de conclusión, hemos practicado un análisis de la implementación de la política pública de expansión del Sistema Universitario de Gestión Estatal en Argentina entre 2003 y 2015, particularmente en el conurbano bonaerense, donde se concentró la creación de instituciones educativas, a fin de evaluar en qué medida la noción de democratización se encontraba presente en las razones declaradas por los decisores de las políticas.

Con relación a los documentos fundacionales de las nuevas universidades, estos describen y ponen en evidencia que efectivamente la creación de instituciones universitarias estuvo motivada por la preocupación de ampliar significativamente el derecho a la educación superior, orientada por la direccionalidad de las políticas sociales y educativas de la etapa que conforman los tres periodos de gobierno kirchneristas, entre 2003 y 2015. En ese contexto, la concepción de la educación superior, como bien público social y deber del Estado y la consideración del derecho a la universidad como derecho humano y universal, conforman el núcleo de sentido de todos los instrumentos que sustentaron la formulación de la política que analizamos. Adicionalmente la declaración acerca de la necesaria intervención del Estado para ampliar las oportunidades de acceso y permanencia en la educación universitaria que resulta favorecedora, en definitiva, de la democratización de la universidad, también forma parte de las motivaciones que declararon los distintos actores que operaron como decisores de la política pública.

A su vez, estudiamos el contenido de la reforma parcial de la Ley de Educación Superior en 2015, que reconoce a la educación como un bien público y un derecho personal y social, en la medida que dicha reforma se inserta en un proceso de democratización universitaria, que el gobierno que asumió en Argentina en 2019 pareciera estar interesado en recuperar.

De acuerdo con lo desarrollado a lo largo de este artículo, creemos que a partir de 2003 se constató la aparición de un nuevo paradigma de políticas para la educación universitaria, del cual formó parte la expansión del Sistema Universitario de Gestión Estatal, con la creación de universidades en toda la extensión del territorio nacional y con mayor impacto en el conurbano bonaerense, cuyo desarrollo fue suspendido con el cambio de gobierno en 2015. Asimismo, del análisis de las líneas de políticas públicas para la educación superior anunciadas por el presidente Fernández, que asumió en 2019 en Argentina, puede aventurarse una suerte de intención de con- 
tinuidad con el proceso de democratización de la universidad impulsado entre 2003 y 2015. Además, ambos gobiernos forman parte del mismo signo político, y quienes protagonizaron el impulso de las dos políticas analizadas integran nuevamente los ámbitos de toma de decisiones de gobierno para el sector en puestos clave. De cualquier modo el escaso tiempo transcurrido no permite ser categórico con la caracterización de la etapa que se inicia. Preferimos ensayar algunos interrogantes sobre el rumbo de las políticas universitarias que desarrollará el nuevo gobierno: ise trata de la reanudación de un ciclo de políticas públicas?; ¿es posible detectar una intención en la continuidad de las políticas de expansión de la educación superior?; ¿ el tono general del discurso sobre las políticas universitarias pondrá el énfasis en la democratización y los derechos?

Sin duda, la democratización de la universidad exigirá compromisos renovados de los actores que intervienen en la formulación e implementación de políticas para el nivel universitario y lograr que el goce y ejercicio del derecho a la universidad se sostenga a lo largo de toda la trayectoria educativa y se aprecie al momento de la graduación de las y los estudiantes. El desafío presente en Argentina pareciera ubicarse no solo en el plano de la necesidad de garantizar la continuidad de las políticas universitarias estatales en un sentido democratizador sino también en el desarrollo de nuevas políticas focalizadas en la trayectoria educativa de los jóvenes que asisten a las universidades. El contexto actual acelera la necesidad de formular e implementar políticas públicas que permitan garantizar el derecho a la universidad en condiciones de igualdad, ya que los efectos económicos de la pandemia acentuarán, sin duda, las desigualdades en el acceso y la permanencia en el nivel superior. Por último, la gran incertidumbre que atraviesa el sector universitario, que al momento de redactar estas páginas, se ha visto obligado a migrar enteramente a una modalidad de educación mediada por tecnologías, exigirá creatividad y capacidad de gestión por parte de los decisores de políticas públicas e institucionales.

\section{Nota}

1. Véanse los discursos de Cristina Fernández de Kirchner en ocasión de la inauguración de la Universidad Nacional del Oeste, del 16/09/2011; de la inauguración de la sede Piñeyro de la Universidad Nacional de Avellaneda, del 13/03/2013 y de la inauguración de la Universidad Nacional Arturo Jauretche del 17/11/2010. 


\section{Referencias}

Basualdo, E. (2006). Estudios de historia económica argentina: desde mediados del siglo $x x$ a la actualidad, Buenos Aires: Siglo Veintiuno Editores.

Basualdo, E. (2011). Sistema político y modelo de acumulación. Tres ensayos sobre la Argentina actual. Buenos Aires: Atuel.

Cantamutto, F. J. (2013). El kirchnerismo como construcción hegemónica populista. Debates urgentes, Año 2, Núm. 3. Buenos Aires.

CENDA (2010). La anatomía del nuevo patrón de crecimiento y la encrucijada actual. La economía argentina en el periodo 2002-2010. Buenos Aires: Atuel.

Chiroleu, A. (2012). Expansión de las oportunidades, inclusión y democratización universitaria. En A. Chiroleu, C. Suasnábar y L. Rovelli, Política universitaria en la Argentina: revisando viejos legados en busca de nuevos horizontes. Los Polvorines: Universidad Nacional de General Sarmiento.

Chiroleu, A. y Iazzetta, O. (2012). La universidad como objeto de política pública durante los gobiernos Kirchner. En A. Chiroleu, M. Marquina y E. Rinesi (compiladores). La política universitaria en los gobiernos Kirchner: continuidades, rupturas, complejidades. Los Polvorines: Universidad Nacional de General Sarmiento.

Ezcurra, A.M. (2011). Igualdad en Educación Superior. Un desafío mundial. Los Polvorines: Universidad Nacional de General Sarmiento. Buenos Aires: IEC- CONADU.

Laguado, A. y Cao, H. (2015). Una administración pública para las sociedades posneoliberales en América Latina (2003-2013), Revista Estado y Políticas Públicas, Núm. 4, Año 3, mayo 2015. Buenos Aires: FLACSO.

Pérez Rasetti, C. (2012). La expansión de la educación universitaria: políticas y lógicas. En A. Chiroleu, M. Marquina y E. Rinesi (compiladores). La política universitaria en los gobiernos Kirchner: continuidades, rupturas, complejidades. Los Polvorines: Universidad Nacional de General Sarmiento.

Rinesi, E. (2015). Filosofía y política de la universidad. Los Polvorines: Universidad Nacional de General Sarmiento. Buenos Aires: IEC-CONADU.

Sidicaro, E. (2003). La crisis del Estado y los actores políticos y socioeconómicos en la Argentina (1989-2001). Buenos Aires: EUDEBA.

Suasnábar, C. y Rovelli, L. (2012). Impensar las políticas universitarias en la Argentina reciente. En A. Chiroleu, M. Marquina y E. Rinesi (compiladores). La política universitaria en los gobiernos Kirchner: continuidades, rupturas, complejidades. Los Polvorines: Universidad Nacional de General Sarmiento.

Unzué, M. (2016). Los sentidos de la idea de la democratización de la universidad en Argentina. En I. Aranciaga (compilador). La universidad y el desafío de construir sociedades inclusivas. Debates y propuestas sobre modelos universitarios desde una perspectiva comparativa. Río Gallegos: Universidad Nacional de la Patagonia Austral.

Varesi, G. (2010). La Argentina post convertibilidad: modelo de acumulación. Problemas del Desarrollo. Revista Latinoamericana de Economía. Vol. 41, Núm. 161, abril-junio. Pp. 141-152. México, disponible en http://www.redalyc.org/ articulo.oa?id=11820105006 (Último ingreso: julio de 2019).

Varesi, G. (2011). Argentina 2002-2011: neodesarrollismo y radicalización progresista. Realidad Económica, Núm. 264, IADE. Buenos Aires. 


\section{Fuentes}

Universidad Nacional Arturo Jauretche (2010). Estatuto provisorio de la Universidad Nacional Arturo Jauretche.

Universidad Nacional Arturo Jauretche (s/f). Proyecto Institucional.

Universidad Nacional de Avellaneda (2015). Estatuto Académico.

Universidad Nacional de Avellaneda (s/f). Proyecto Institucional Universitario de la UNDAV.

Universidad Nacional de José C. Paz (2015). Estatuto provisorio de la Universidad Nacional de José C. Paz.

Universidad Nacional de Moreno (2010). Proyecto Institucional.

Universidad Nacional de Moreno (2013). Estatuto de la Universidad Nacional de Moreno.

Universidad Nacional del Oeste (2013). Estatuto.

\section{Normativa}

Ley de implementación efectiva de la responsabilidad del Estado en el nivel de Educación Superior, Ley 27.204.

Universidad Nacional de Avellaneda, Ley 26.543.

Universidad Nacional del Oeste, Ley 26.544.

Universidad Nacional de Moreno, Ley 26.575.

Universidad Nacional Arturo Jauretche, Ley 26.576.

Universidad Nacional de José Clemente Paz, Ley 26.577.

Q6 Universidades núm. 85, julio-septiembre 2020 UDUAL DOI:https://doi.org/10.36888/udual.universidades.2020.85.278

Anabella Celeste Lucardi $\quad$ Las políticas universitarias del nuevo gobierno en Argentina: el desafío de fortalecer la democratización de la Universidad 